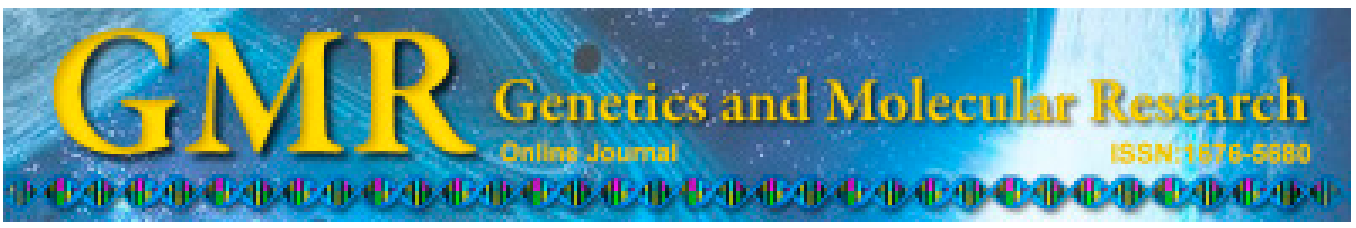

\title{
Serum level of endothelial cell-specific molecule-1 and prognosis of colorectal cancer
}

\author{
H. Jiang, X.G. Fu and Y.T. Chen \\ Department of Colorectal Surgery, Binzhou Medical University Hospital, \\ Binzhou, China \\ Corresponding author: H. Jiang \\ E-mail: dr_jianghong@126.com \\ Genet. Mol. Res. 14 (2): 5519-5526 (2015) \\ Received June 23, 2014 \\ Accepted November 4, 2014 \\ Published May 25, 2015 \\ DOI http://dx.doi.org/10.4238/2015.May.25.3
}

\begin{abstract}
We evaluated the clinical significance and explored the prognostic value of serum endothelial cell-specific molecule-1 (ESM-1) expression in colorectal cancer (CRC) in a Chinese population. Serum samples were obtained from 89 CRC patients undergoing surgical treatment and 90 healthy volunteers (control group). ESM-1 levels in serum samples from CRC patients and controls were measured using a sandwich enzyme-linked immunosorbent assay. Overall survival was analyzed by the log-rank test, and survival curves were plotted according to the Kaplan-Meier method. Univariate Cox regression was performed on each clinical covariate to examine its influence on patient survival. Final multivariate models were based on step-wise addition. Serum ESM-1 expression levels were significantly higher in patients with CRC $(66.81 \pm 22.97 \mathrm{pg} / \mathrm{mL})$ than in healthy volunteers $(31.50 \pm$ $16.81 \mathrm{pg} / \mathrm{mL}, \mathrm{P}=0.012$ ). Expression levels of ESM-1 in CRC patients were significantly correlated with histological differentiation $(\mathrm{P}=$ 0.004), TNM stage $(P=0.007)$, depth of tumor invasion $(P=0.02)$, and lymph node metastasis $(\mathrm{P}=0.001)$. Kaplan-Meier survival curves revealed that the overall survival was significantly lower in patients with high ESM-1 levels than in those with low levels (29.43 vs $61.20 \%$, $\mathrm{P}=0.0166$ ). Multivariate analysis confirmed that the hazard risk of
\end{abstract}


death was significantly higher in patients with high serum ESM-1 expression compared with low expression (hazard risk $=4.09,95 \%$ confidence interval: 2.27-10.88, $\mathrm{P}<0.001$ ). Detection of ESM-1 levels in the serum may serve as a tumor biomarker for assessing prognosis in $\mathrm{CRC}$.

Key words: Colorectal cancer; Endothelial cell-specific molecule-1; Prognosis; Marker

\section{INTRODUCTION}

Colorectal cancer (CRC) is the third most commonly diagnosed cancer worldwide in males and second in females, with over 1.2 million new cases diagnosed in 2008 and accounting for $9.7 \%$ of all incident cancers (Siegel et al., 2014). The exact mechanism responsible for CRC development is unknown (Akhtar et al., 2014; Fujii and Sato, 2014). Despite the use of multimodel treatment strategies, including surgery, perioperative chemotherapy, radiotherapy, and targeted therapy, CRC is still the second leading cause of cancer-related death in Western countries (Weitz et al., 2005). Therefore, there is a crucial need to identify novel cancer-related genes for predicting the progression and prognosis of CRC as well as to help establish treatment strategies.

Endothelial cell-specific molecule-1 (ESM-1), also known as endocan, is a dermatan sulfate proteoglycan that was initially cloned in 1996 from a human endothelial cell cDNA library (Lassalle et al., 1996; Béchard et al., 2001). ESM-1 is a 50-kDa cysteine-rich proteoglycan, of which approximately $30 \mathrm{kDa}$ corresponds to a single dermatan sulfate chain (Lassalle et al., 1996; Béchard et al., 2001; Sarrazin et al., 2006). Previous studies have shown that ESM-1 is secreted by vascular endothelial cells, epithelial cells lining renal distal tubules, bronchi, and lung submucosal glands (Béchard et al., 2000; Abid et al., 2006). ESM-1 is overexpressed in the vascular endothelium of renal cell carcinoma, breast carcinoma, glioma, nonsmall cell lung cancer, and CRC (Aitkenhead et al., 2002; Grigoriu et al., 2006; Scherpereel et al., 2006). Previously, Ji et al. (2010) found that expression of ESM-1 was increased in the serum of CRC patients and that ESM-1 may be used as a serum marker for the early detection of CRC; furthermore, higher serum ESM-1 level was correlated with poor prognosis. However, the association between the serum level of ESM-1 and the prognosis of CRC patients has not been investigated in the Chinese population. In this study, we examined the clinical significance and explored the prognostic value of serum ESM-1 expression level in CRC in a Chinese population.

\section{MATERIAL AND METHODS}

\section{Patients and blood specimens}

We collected preoperative blood specimens from 89 patients diagnosed with CRC (51 males and 38 females; age range, 31-88 years) who had not undergone any form of preoperative chemotherapy or radiation therapy at the Department of Colorectal Surgery, Binzhou Medical University Hospital, Binzhou, China between March 2008 and June 2013. Furthermore, patients who suffered from other cancers were excluded. Patients were classified ac- 
cording to the tumor-node-metastasis (TNM) classification of the International Union against Cancer. The control group included peripheral blood specimens collected from 90 healthy volunteers (52 males and 38 females; age range, 29-74 years) who had never received a diagnosis of malignancy. The clinicopathological parameters of CRC patients are shown in Table 1. Serum samples were obtained after centrifugation from peripheral venous blood and immediately stored at $-80^{\circ} \mathrm{C}$ until use. Before enrollment, this study protocol was approved by the ethics committee of Binzhou Medical University Hospital. Informed consent was obtained from each of the recruited patients and health volunteers.

\begin{tabular}{|c|c|c|c|}
\hline Clinicopathological factors & No. of patients & Serum ESM-1 level (pg/mL, Means \pm SD) & $P$ value \\
\hline \multicolumn{4}{|l|}{ Age (years) } \\
\hline$\geq 65$ & 47 & $69.22 \pm 26.21$ & \multirow[t]{2}{*}{0.34} \\
\hline$<65$ & 42 & $60.68 \pm 20.92$ & \\
\hline \multicolumn{4}{|l|}{ Gender } \\
\hline Male & 51 & $67.75 \pm 27.25$ & \multirow[t]{2}{*}{0.78} \\
\hline Female & 38 & $65.76 \pm 20.26$ & \\
\hline \multicolumn{4}{|l|}{ Tumor site } \\
\hline Colon & 49 & $66.97 \pm 23.46$ & \multirow[t]{2}{*}{0.81} \\
\hline Rectum & 40 & $66.16 \pm 20.17$ & \\
\hline \multicolumn{4}{|l|}{ Histology } \\
\hline Well & 34 & $44.17 \pm 18.28$ & \multirow[t]{2}{*}{0.004} \\
\hline Moderate/poor (mucinous) & 55 & $79.92 \pm 29.11$ & \\
\hline \multicolumn{4}{|l|}{ TNM stage } \\
\hline $\mathrm{I} / \mathrm{II}$ & 48 & $47.56 \pm 19.29$ & \multirow[t]{2}{*}{0.007} \\
\hline III/IV & 41 & $77.29 \pm 28.24$ & \\
\hline \multicolumn{4}{|l|}{ Tumor size } \\
\hline$\geq 5 \mathrm{~cm}$ & 46 & $72.11 \pm 26.23$ & \multirow[t]{2}{*}{0.06} \\
\hline$<5 \mathrm{~cm}$ & 43 & $55.28 \pm 18.21$ & \\
\hline \multicolumn{4}{|l|}{ Depth of tumor invasion } \\
\hline $\mathrm{T} 1 / 2$ & 50 & $48.12 \pm 17.29$ & \multirow[t]{2}{*}{0.02} \\
\hline $\mathrm{T} 3 / 4$ & 39 & $74.29 \pm 21.98$ & \\
\hline \multicolumn{4}{|l|}{ Lymph node metastasis } \\
\hline Yes & 44 & $80.27 \pm 26.28$ & \multirow[t]{2}{*}{0.001} \\
\hline No & 45 & $42.18 \pm 18.29$ & \\
\hline \multicolumn{4}{|l|}{ Serum CEA level $(\mu \mathrm{g} / \mathrm{L})$} \\
\hline$\geq 5$ & 52 & $71.98 \pm 21.77$ & \multirow[t]{2}{*}{0.09} \\
\hline$<5$ & 37 & $59.17 \pm 27.97$ & \\
\hline
\end{tabular}

\section{Sandwich enzyme-linked immunosorbent assay (ELISA) for ESM-1}

The ESM-1 ELISA kits were obtained from Atila Biosystems (Palo Alto, CA, USA) and used to detect ESM-1 according to manufacturer instructions. All samples were coded using a numbering system and were analyzed by technicians in our laboratory who were not aware of the disease state of the patients. A microwell plate was coated with $100 \mu \mathrm{L}$ capture antibody (1 $\mu \mathrm{g} / \mathrm{mL}$ ) against ESM-1, incubated overnight at $4^{\circ} \mathrm{C}$, and then coated again with $1 \%$ bovine serum albumin. Next, $100 \mu \mathrm{L}$ serially diluted ESM-1 standard solution and samples diluted 5-fold in dilution buffer ( $2 \%$ bovine serum albumin in phosphate-buffered saline containing Tween 20) were added to wells in duplicate and incubated with $0.25 \mu \mathrm{g} / \mathrm{mL}$ detection antibody for $1 \mathrm{~h}$ at room temperature. After washing the wells with phosphate-buffered saline containing Tween 20, $100 \mu \mathrm{L}$ diluted horseradish peroxidase-conjugated strepatvidin was added to each well and incubated for $30 \mathrm{~min}$. Subsequently, tetramethylbenzidine solution was added to the wells, and 
the reaction was stopped using $1 \mathrm{~N} \mathrm{H}_{2} \mathrm{SO}_{4}$ solution. Absorbance was then measured at $450 \mathrm{~nm}$ using a spectrophotometer (Molecular Devices, Sunnyvale, CA, USA).

\section{Statistical analysis}

Statistical analyses were performed using the SPSS 18.0 software (Chicago, IL, USA) and the GraphPad Prism 5 (GraphPad Software Inc., CA, USA). Numerical variables were recorded as means \pm standard deviation and analyzed by independent Student $t$-tests. Categorical variables are presented as rates and were analyzed using the chi-square test or Fisher's exact test. Overall survival was analyzed by the log-rank test, and survival curves were plotted according to the Kaplan-Meier method. Univariate Cox regression was performed on each clinical covariate to examine its influence on patient survival. Final multivariate models were based on step-wise addition. P-values $<0.05$ were considered to be statistically significant.

\section{RESULTS}

\section{Comparison of serum ESM-1 expression levels between CRC patients and healthy controls}

A sandwich ELISA was used to detect the expression levels of serum ESM-1 from 89 patients with CRC and 90 healthy volunteers. As shown in Figure 1, serum ESM-1 expression levels were significantly higher in patients with CRC $(66.81 \pm 22.97 \mathrm{pg} / \mathrm{mL})$ than those in healthy volunteers $(31.50 \pm 16.81 \mathrm{pg} / \mathrm{mL}, \mathrm{P}=0.012)$.

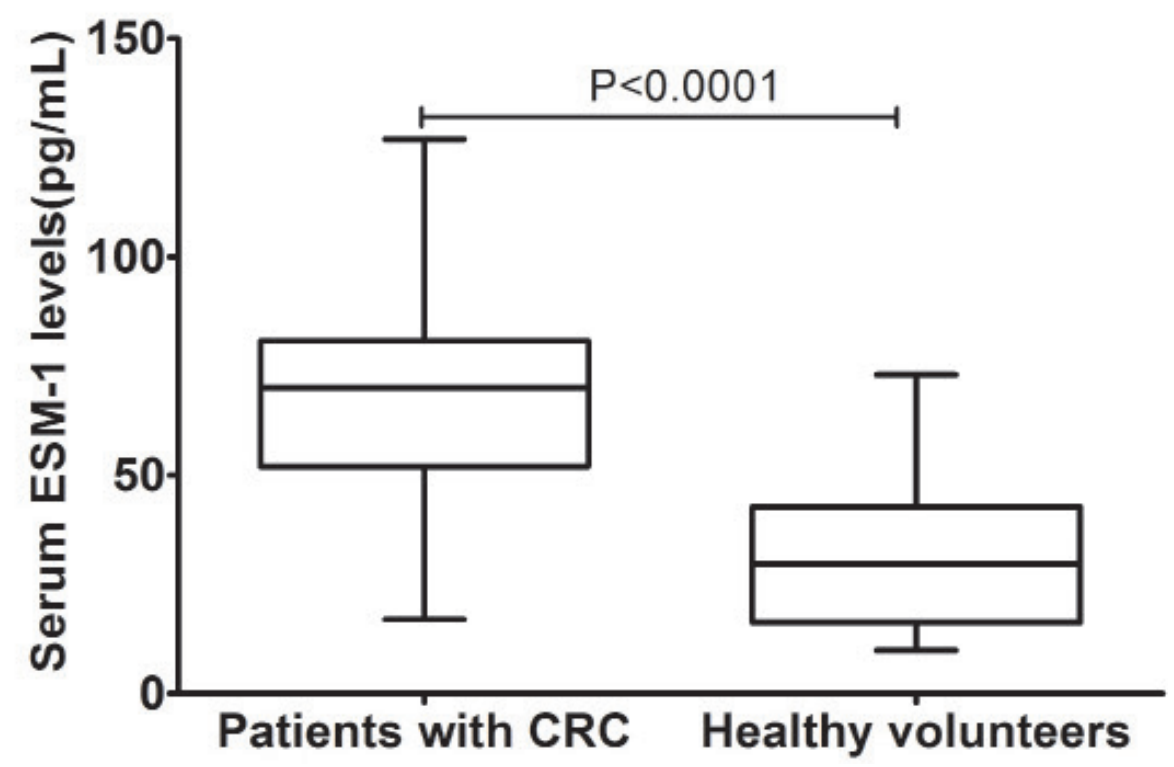

Figure 1. Serum ESM-1 levels in patients with CRC and healthy participants. Significantly elevated ESM-1 levels were observed in patients with CRC compared with those in healthy controls $(\mathrm{P}<0.0001)$. 


\section{Correlations between serum ESM-1 expression levels and clinicopathological features of CRC patients}

In order to determine the clinical significance of ESM-1, we correlated the ESM-1 expression levels with patients' clinicopathological features (Table 1). We found that the expression levels of ESM-1 in patients with CRC were significantly correlated with histological differentiation $(\mathrm{P}=0.004)$, TNM stage $(\mathrm{P}=0.007)$, depth of tumor invasion $(\mathrm{P}=0.02)$, and lymph node metastasis $(\mathrm{P}=0.001)$. However, no statistically significant correlations between ESM-1 expression levels and gender, age, tumor location, tumor size, or serum carcinoembryonic antigen (CEA) level were observed (all $\mathrm{P}>0.05)$.

\section{Relationship between serum ESM-1 expression levels and survival in 89 CRC patients}

Based on the median value of serum ESM-1 levels, we selected a cut-off value of $68.4 \mathrm{pg} / \mathrm{mL}$ to divide the patients into 2 groups with low $(\mathrm{N}=44)$ and high $(\mathrm{N}=45)$ ESM-1 levels. Kaplan-Meier survival curves demonstrated that the overall survival rate was significantly lower in the patients with high levels of ESM-1 than in those with low levels (29.43 vs $61.20 \%, \mathrm{P}=0.0166$, Figure 2). As shown in Table 2, multivariate analysis confirmed that the hazard risk of death was significantly higher in patients with high serum expression levels of ESM-1 compared with low expression levels (hazard risk $=4.09,95 \%$ confidence interval: $2.27-10.88, \mathrm{P}<0.001)$. In addition, a higher TNM stage $(\mathrm{P}=0.002)$ was correlated with poorer histological differentiation $(\mathrm{P}=0.006)$, and lymph node metastasis $(\mathrm{P}=0.03)$ was also significantly associated with an elevated hazard risk of death in CRC patients.

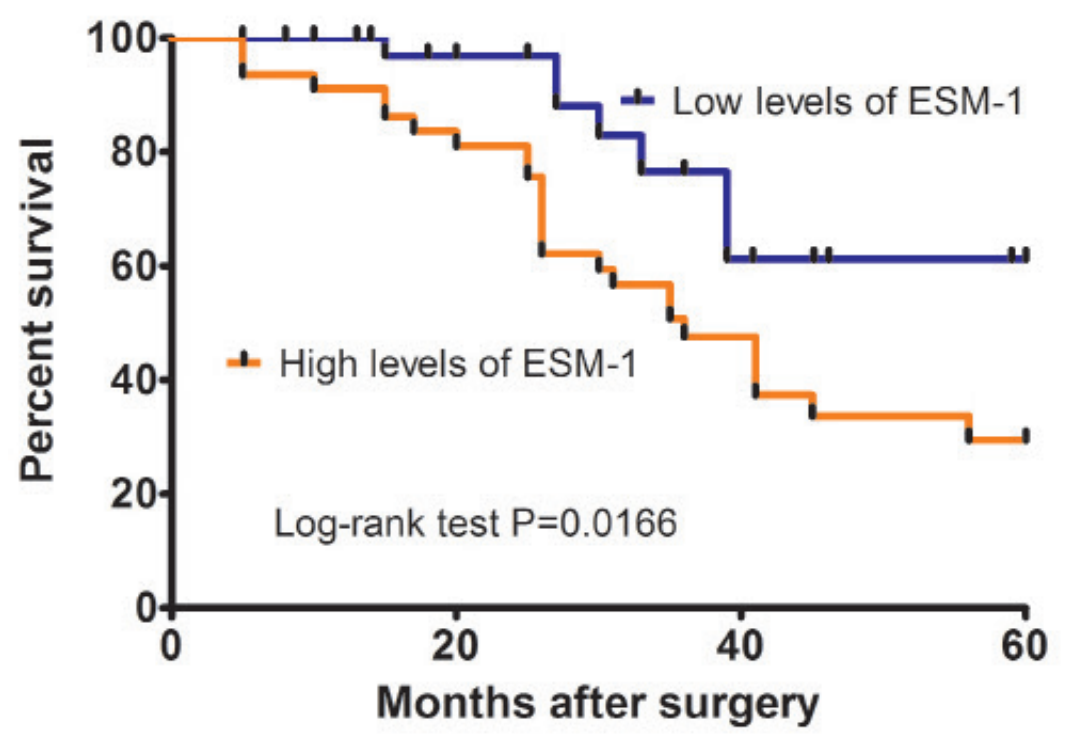

Figure 2. Kaplan-Meier analysis for the correlation between serum ESM-1 levels and survival. Overall survival was significantly lower in the patients with high levels of ESM-1 than in those with low levels $(29.43 v s 61.20 \%$, $\mathrm{P}=0.0166)$. 
Table 2. Multivariate analysis of the associations of prognosis with various clinicopathologic parameters and serum ESM-1 expression in 89 CRC patients.

\begin{tabular}{llcc}
\hline Features & HR & $95 \%$ CI & P value \\
\hline Age & 1.21 & $0.48-3.89$ & 0.25 \\
Gender & 0.98 & $0.23-2.91$ & 0.78 \\
Tumor site & 2.38 & $0.79-5.76$ & 0.09 \\
Histology & 3.47 & $2.71-8.01$ & 0.006 \\
TNM stage & 3.87 & $2.98-8.29$ & 0.002 \\
Tumor size & 1.89 & $0.71-6.22$ & 0.07 \\
Depth of tumor invasion & 2.85 & $0.93-7.11$ & 0.06 \\
Lymph node metastasis & 3.91 & $1.92-8.19$ & 0.03 \\
Serum CEA level & 2.17 & $0.81-3.91$ & 0.09 \\
Serum ESM-1 level & 4.09 & $2.27-10.88$ & $<0.001$ \\
\hline
\end{tabular}

$\mathrm{HR}=$ hazards ratio; $\mathrm{CI}=$ confidence interval.

\section{DISCUSSION}

$\mathrm{CRC}$ is one of the leading causes of cancer-related deaths worldwide. Although there has been great progress in the diagnosis and prognosis of CRC over the past several decades, early detection screening methods must be improved and new prognostic biomarkers should be identified. Previously, Ji et al. (2010) found that expression of ESM-1 was increased in the serum of CRC patients and that ESM-1 can be used as a potential serum marker for the early detection of CRC. They showed that the ESM-1 levels in the serum of patients with CRC were significantly elevated $(70.1 \pm 29.7 \mathrm{pg} / \mathrm{mL})$ compared to those in healthy subjects $(29.7$ $\pm 14.9 \mathrm{pg} / \mathrm{mL}$ ). The accuracy, sensitivity, and specificity of ESM-1 for predicting CRC were $0.94,99$, and $73 \%$, respectively, according to receiver operating characteristics curve analysis. The positive predictive value and negative predictive value were 63 and $95 \%$, respectively. The likelihood ratios of a positive or negative test result were 73 and 0.27 , respectively. When analyzed by Cox regression analysis, a higher serum ESM-1 level $(\geq 76.0 \mathrm{pg} / \mathrm{mL})$ was correlated with poor prognosis. However, the association between the serum level of ESM-1 and the prognosis of CRC patients has not been investigated in Chinese populations. In the present study, we examined the clinical significance and prognostic value of serum ESM-1 expression level in CRC in a Chinese population. We found that serum ESM-1 expression levels were significantly higher in patients with $C R C$ than in healthy volunteers $(P=0.012)$. The expression levels of ESM-1 in patients with CRC were significantly correlated with histological differentiation, TNM stage, depth of tumor invasion, and lymph node metastasis. Kaplan-Meier survival curves revealed that the overall survival rate was significantly lower in the patients with high levels of ESM-1 than in those with low levels $(\mathrm{P}=0.0166)$. Multivariate analysis confirmed that the hazard risk of death was significantly higher in patients with high serum expression levels of ESM-1 compared with low expression levels. These findings imply that serum ESM-1 level is involved in the development and metastasis of cancer, and may have prognostic significance in CRC.

ESM-1 is a $50-\mathrm{kDa}$ secretory proteoglycan comprised of a mature polypeptide of 165 amino acids and a single dermatan sulfate chain covalently linked to the serine residue at position 137 (Sarrazin et al., 2006). It is a key player in the regulation of cell adhesion, inflammatory disorders, and tumor progression. ESM-1 specifically associates with insulin-like growth factor to promote cell growth and proliferation, and its expression is induced by tumor 
necrosis factor- $\alpha$ and interleukin- $1 \beta$ (Lassalle et al., 1996; Sarrazin et al., 2006). It is expressed by the vascular endothelium, freely circulates in the bloodstream, and has only 1 glycosaminoglycan chain (Sarrazin et al., 2006). It was recently reported that ESM-1 is expressed in the tumor endothelium and induces tumor formation (Aitkenhead et al., 2002; Scherpereel et al., 2003). Levels of ESM-1 are increased in vascular endothelial growth factor- $\alpha$ treated endothelial cells and in human renal clear cell carcinoma (Rennel et al., 2007). High expression of ESM-1 has been shown to be correlated with poor prognosis and metastasis in several types of human cancers, including breast, renal, lung, liver, and brain cancer (Sarrazin et al., 2006; Huang et al., 2009; Maurage et al., 2009). In addition, serum ESM-1 level was correlated with survival and time to tumor progression in lung cancer as well as gastric cancer (Grigoriu et al., 2006; Sun et al., 2014). Grigoriu et al. (2006) evaluated the expression of ESM-1 in non-small cell lung tumors compared with normal lungs and studied the significance of high levels of circulating ESM-1 in patients with non-small cell lung cancer. They demonstrated that ESM-1 was overexpressed in non-small cell lung tumors compared with healthy lungs and likely represents the response of the tumoral endothelium to proangiogenic growth factor stimulation. Circulating levels of ESM-1 may reflect tumor angiogenic stimulation and be of prognostic significance. Sun et al. (2014) found that compared to healthy volunteers, serum ESM-1 levels in gastric cancer patients were increased $(\mathrm{P}<0.01)$. The sensitivity and specificity of serum ESM-1 were 73.9 and 51.2\%, respectively. In contrast, the sensitivities of CEA, CA19-9, and CA72-4 were only 16.1, 18.3, and 23.2\% respectively. In addition, high level of serum ESM-1 indicated poor outcomes $(\mathrm{P}<0.05)$. Therefore, serum ESM-1 level may be a potential serum marker for the diagnosis and prediction of prognosis of gastric cancer patients (Sun et al., 2014). Our findings are consistent with the findings of the previous study. A limitation of the present study was the small number of patients examined. Further studies including larger sample numbers are needed to confirm whether serum ESM-1 level can be used as a prognostic marker for CRC.

In conclusion, we found that serum ESM-1 levels were significantly elevated in patients with CRC compared with in control subjects and that the overall survival rate was significantly lower in patients with high levels of ESM-1 than in those with low levels. These findings indicate that serum ESM-1 level can be used as a novel prognostic marker of CRC.

\section{Conflicts of interest}

The authors declare no conflict of interest.

\section{REFERENCES}

Abid MR, Yi X, Yano K, Shih SC, et al. (2006). Vascular endocan is preferentially expressed in tumor endothelium. Microvasc. Res. 72: 136-145.

Aitkenhead M, Wang SJ, Nakatsu MN, Mestas J, et al. (2002). Identification of endothelial cell genes expressed in an in vitro model of angiogenesis: induction of ESM-1, (beta)ig-h3, and NrCAM. Microvasc. Res. 63: 159-171.

Akhtar R, Chandel S, Sarotra P and Medhi B (2014). Current status of pharmacological treatment of colorectal cancer. World J. Gastrointest. Oncol. 6: 177-183.

Béchard D, Meignin V, Scherpereel A, Oudin S, et al. (2000). Characterization of the secreted form of endothelial-cellspecific molecule 1 by specific monoclonal antibodies. J. Vasc. Res. 37: 417-425.

Béchard D, Gentina T, Delehedde M, Scherpereel A, et al. (2001). Endocan is a novel chondroitin sulfate/dermatan sulfate proteoglycan that promotes hepatocyte growth factor/scatter factor mitogenic activity. J. Biol. Chem. 276: 4834148349 . 
Fujii M and Sato T (2014). Culturing intestinal stem cells: applications for colorectal cancer research. Front. Genet. 5: 169. Grigoriu BD, Depontieu F, Scherpereel A, Gourcerol D, et al. (2006). Endocan expression and relationship with survival in human non-small cell lung cancer. Clin. Cancer Res. 12: 4575-4582.

Huang GW, Tao YM and Ding X (2009). Endocan expression correlated with poor survival in human hepatocellular carcinoma. Dig. Dis. Sci. 54: 389-394.

Ji NY, Kim YH, Jang YJ, Kang YH, et al. (2010). Identification of endothelial cell-specific molecule-1 as a potential serum marker for colorectal cancer. Cancer Sci. 101: 2248-2253.

Lassalle P, Molet S, Janin A, Heyden JV, et al. (1996). ESM-1 is a novel human endothelial cell-specific molecule expressed in lung and regulated by cytokines. J. Biol. Chem. 271: 20458-20464.

Maurage CA, Adam E, Mineo JF, Sarrazin S, et al. (2009). Endocan expression and localization in human glioblastomas. J. Neuropathol. Exp. Neurol. 68: 633-641.

Rennel E, Mellberg S, Dimberg A, Petersson L, et al. (2007). Endocan is a VEGF-A and PI3K regulated gene with increased expression in human renal cancer. Exp. Cell Res. 313: 1285-1294.

Sarrazin S, Adam E, Lyon M, Depontieu F, et al. (2006). Endocan or endothelial cell specific molecule-1 (ESM-1): a potential novel endothelial cell marker and a new target for cancer therapy. Biochim. Biophys. Acta 1765: 25-37.

Scherpereel A, Gentina T, Grigoriu B, Sénéchal S, et al. (2003). Overexpression of endocan induces tumor formation. Cancer Res. 63: 6084-6089.

Scherpereel A, Depontieu F, Grigoriu B, Cavestri B, et al. (2006). Endocan, a new endothelial marker in human sepsis. Crit. Care Med. 34: 532-537.

Siegel R, DeSantis C and Jemal A (2014). Colorectal cancer statistics, 2014. CA Cancer J. Clin. 64: 104-117.

Sun W, Zhang L, Cheng X, Wang X, et al. (2014). Detection of serum endothelial cell specific molecule 1 in gastric cancer patients and its clinical significance. Zhonghua Wei Chang Wai Ke Za Zhi 17: 51-55.

Weitz J, Koch M, Debus J, Höhler T, et al. (2005). Colorectal cancer. Lancet 365: 153-165. 